\title{
Elastoplastic behavior identification for heterogeneous loadings and materials
}

\author{
F. Latourte, A. Chrysochoos, S. Pagano, B. Wattrisse
}

\begin{abstract}
Image processing techniques give access to full field measurements of different thermomechanical data (strain, strain-rate [1], temperature [2], ...). These techniques have become more and more useful for obtaining a fine and local description of material properties. As they allow to measure complete thermal and mechanical fields, they can be used to identify several parameters of the constitutive equations during a single deformation process using specifically designed heterogeneous tests [3].

In [4], surface strain fields obtained by Digital Image Correlation were used to identify the distribution of elastic parameters and stress fields by minimizing a given energy functional.

In this paper, the previous method is improved by a relevant choice for stress approximation, and then extended to a wider class of elastoplastic materials. Its reliability is then checked through applications on simulated data obtained under small perturbations and plane stress assumptions. In particular, the robustness of the method with respect to measurement noise is studied on the basis on numerical data. An experimental application to heterogeneous material identification is, finally, proposed.
\end{abstract}

\section{Introduction}

During standard mechanical tests (simple tension, simple shear), the response of the specimen gauge part is generally associated with the material response. Nevertheless, the unavoidable defects of experimental set-up, of sample geometry or of the material may result in a heterogeneous response of the gauge part. In order to take this matter of fact into account, mechanical tests are now often coupled with full field measurement techniques (displacement, strain, temperature,...) that better reflect the local behavior of the material.

The work presented hereafter aims at capitalizing on the richness of data provided by these techniques to locally identify the material properties during mechanical tests. The identification of material properties from field measurements is a particularly active research area that has lead to the development of various approaches, with some of the most widespread being : the Finite Element Model Updating method [5], the Virtual Field method [6], or the Equilibrium Gap Method [7].

A variational method due to Kohn et al. [8], initially developed for the identification of electrical conductivities was extended to the identification of both elastic parameters and stress distribution by writing a functional expressing the constitutive equation gap [4]. In this paper, we generalize this latter approach to the case of elasto-plastic materials with linear kinematic hardening in the context of a small perturbations hypothesis and plane stress assumption for quasi-static loadings. The displacement fields are provided by the Digital Image Correlation (D.I.C.) method detailed in [1].

In a first part, we present the theoretical backgrounds and the numerical implementation of the identification procedure. In the second part, the performances of the method are tested on the basis of numerical data corresponding to a tensile test performed on a bi-material sample. We will particularly focus on its ability to catch the material interfaces and check its robustness with respect to measurement noise. Finally, we discuss the results of an identification of a kinematic elastoplastic model carried out on experimental data obtained in a similar situation. 


\section{Identification procedure}

The material behavior is locally identified from in plane displacement distributions obtained by D.I.C. In the most general case, these surface measurements do not allow to identify material properties on a whole volume. For that reason, we restrict our attention to thin flat samples for which the plane stress assumption remains realistic. For each deformation increment, the local measurements of three in-plane strain components allow us to locally determine three material parameters at the most. Here, we introduce the framework chosen to state and solve the identification problem associated with an elastoplastic behavior with positive hardening under the small perturbation hypothesis.

This model is expressed as follows [9]:

$$
\begin{aligned}
\boldsymbol{\sigma} & =\mathbf{A}_{e}\left(\boldsymbol{\varepsilon}-\boldsymbol{\varepsilon}_{p}\right) \\
f(\boldsymbol{\sigma}, \mathbf{X}) & =(\boldsymbol{\sigma}-\mathbf{X})_{I I}-\sigma_{0} \leq 0 \\
\dot{\varepsilon_{p}} & =\dot{\gamma} \frac{\partial f}{\partial \boldsymbol{\sigma}} \\
\dot{\mathbf{X}} & =\frac{2}{3} k \dot{\varepsilon_{p}}
\end{aligned}
$$

where $\mathbf{A}_{e}$ is the elasticity tensor, $\boldsymbol{\sigma}$ the stress, $\boldsymbol{\varepsilon}$ the strain, $\boldsymbol{\varepsilon}_{p}$ the plastic strain, $\gamma$ the plastic multiplier, $f$ is the yield function, $\sigma_{0}$ the yield stress, $\mathbf{X}$ the backstress and $k$ the hardening modulus. $(\cdot)_{I I}$ stands for the von Mises norm.

\subsection{Theoretical backgrounds}

For a sequence of successive loadsteps, the in-plane displacement field $\mathbf{u}^{*}$ is measured on a given region of interest $\Omega$ of a specimen. The overall forces $\mathbf{R}_{i}$ are given on some parts $\Gamma_{i}$ of the boundary of $\Omega$ and the stress-free parts $\Gamma_{j}$ are such that $\Gamma_{i} \cup \Gamma_{j}=\partial \Omega$ and $\Gamma_{i} \cap \Gamma_{j}=\oslash$. The density of the volume force $f$ is here considered to be equal to zero.

Starting from an experimental set of images, it seems particularly promising to use two different descriptions of the mechanical problem, one based on the total displacement field $\mathbf{u}^{*}$, which is referred to here as "standard formulation" and another one referred to as "incremental formulation", based on an incremental displacement field $\Delta \mathbf{u}^{*}$ between two images:

$$
\begin{aligned}
& \text { Standard formulation } \\
& \text { div } \boldsymbol{\sigma}=0 \text { in } \Omega \\
& \boldsymbol{\sigma}=\mathbf{A}^{s} \boldsymbol{\varepsilon}\left(\mathbf{u}^{*}\right) \text { in } \Omega \\
& \left\{\begin{array}{l}
\mathbf{R}_{i}=\int_{\Gamma_{i}} \boldsymbol{\sigma} \cdot \mathbf{n} d s \\
\boldsymbol{\sigma} \cdot \mathbf{n}=0 \text { on } \Gamma_{j}
\end{array}\right.
\end{aligned}
$$

Incremental formulation

$$
\begin{aligned}
& \operatorname{div} \Delta \boldsymbol{\sigma}=0 \quad \text { in } \Omega \\
& \Delta \boldsymbol{\sigma}=\mathbf{A}^{t} \boldsymbol{\varepsilon}\left(\Delta \mathbf{u}^{*}\right) \quad \text { in } \Omega \\
& \left\{\begin{array}{l}
\Delta \mathbf{R}_{i}=\int_{\Gamma_{i}} \Delta \boldsymbol{\sigma} \cdot \mathbf{n} d s \\
\Delta \boldsymbol{\sigma} \cdot \mathbf{n}=0 \quad \text { on } \Gamma_{j}
\end{array}\right.
\end{aligned}
$$

where $\mathbf{A}^{s}$ and $\mathbf{A}^{t}$ stand for the secant and tangent stiffness tensor respectively, $\boldsymbol{\sigma}$ and $\Delta \boldsymbol{\sigma}$ represent the stress field and the incremental stress field respectively, and $\varepsilon\left(\mathbf{u}^{*}\right)$ and $\varepsilon\left(\Delta \mathbf{u}^{*}\right)$ are the strain related to the measured displacement and to the incremental one, respectively. An additive decomposition of the strain tensor into an elastic and a plastic part is assumed in the following. For a linear kinematic model, the tensors $\mathbf{A}^{s}$ and $\mathbf{A}^{t}$ can be expressed directly as a function of the material parameters and of the loading history ( $c f$. section 2.2.3). A couple $\left(\mathbf{A}^{s}, \boldsymbol{\sigma}\right)$ is a solution to the standard identification problem if it satisfies the equilibrium equation (5), the constitutive equation (6) and the global equilibrium (7). Respectively, a couple $\left(\mathbf{A}^{t}, \Delta \boldsymbol{\sigma}\right)$ is a solution to the incremental identification problem if it satisfies equations $(8,9,10)$, where $\Delta \mathbf{R}_{i}$ represents the reaction increment on the boundary $\Gamma_{i}$. 


\subsubsection{Defining a constitutive equation gap functional}

Our elastoplastic identification problem is to characterize the mechanical behavior within the $\Omega$ domain. Inside the elastic domain, the behavior can be described by an elastic stiffness matrix $\mathbf{B}_{e}$, obtained either by the "standard" or the "incremental formulation". Indeed, from a theoretical point of view $\mathbf{B}_{e}=\mathbf{B}_{e}^{s}=\mathbf{B}_{e}^{t}$, where $\mathbf{B}_{e}^{s}$ is the elastic secant tensor and $\mathbf{B}_{e}^{t}$ the elastic tangent tensor.

Once plasticity occurs, the history-dependent behavior can be described either by a plastic tangent matrix $\mathbf{B}_{p}^{t}$ or by a plastic secant matrix $\mathbf{B}_{p}^{s}$ both depending on the plastic parameters associated with the model chosen. As will be shown later, the "standard formulation" is used to obtain $\mathbf{B}_{e}^{s}$ or $\mathbf{B}_{p}^{s}$ while the "incremental formulation" allows to identify either $\mathbf{B}_{e}^{t}$ or $\mathbf{B}_{p}^{t}$, or to check the presence of elastic unloadings.

We associate the constitutive equation gap functional $\mathcal{F}$ to the "standard formulation" and the functional $\mathcal{G}$ to the "incremental formulation":

$$
\begin{aligned}
& \mathcal{F}\left(\boldsymbol{\tau}, \mathbf{B}^{s}\right)=\frac{1}{2} \int_{\Omega}\left(\boldsymbol{\tau}-\mathbf{B}^{s}: \varepsilon\left(\mathbf{u}^{*}\right)\right)^{T}: \mathbf{B}^{s-1}:\left(\boldsymbol{\tau}-\mathbf{B}^{s}: \varepsilon\left(\mathbf{u}^{*}\right)\right) d \Omega \\
& \mathcal{G}\left(\Delta \boldsymbol{\tau}, \mathbf{B}^{t}\right)=\frac{1}{2} \int_{\Omega}\left(\Delta \boldsymbol{\tau}-\mathbf{B}^{t}: \varepsilon\left(\Delta \mathbf{u}^{*}\right)\right)^{T}: \mathbf{B}^{t-1}:\left(\Delta \boldsymbol{\tau}-\mathbf{B}^{t}: \varepsilon\left(\Delta \mathbf{u}^{*}\right)\right) d \Omega
\end{aligned}
$$

The two stress fields $\boldsymbol{\tau}$ and $\Delta \boldsymbol{\tau}$ belong respectively to spaces $\Sigma_{a d m}$ and $\Delta \Sigma_{a d m}$ :

$$
\begin{aligned}
\Sigma_{a d m} & =\{\boldsymbol{\tau} \text { regular and symmetric satisfying the eqs. (5) and (7) }\} \\
\Delta \Sigma_{a d m} & =\{\Delta \boldsymbol{\tau} \text { regular and symmetric satisfying the eqs. (8) and (10) }\}
\end{aligned}
$$

Under the plane-stress assumption and using engineering notations, let $\xi_{e}$ be the admissible elastic stiffness tensor space and, in the case of positive hardening, $\xi_{p}$ the admissible plastic stiffness tensor space:

$$
\begin{aligned}
& \xi_{e}=\left\{\mathbf{B}_{e} \in\left(\mathcal{L}^{\infty}(\Omega)\right)_{s y m}^{3 \times 3} ; B_{i j} \text { piecewise linear, } \mathbf{B}_{e} \text { non negative definite }\right\} \\
& \xi_{p}=\left\{\mathbf{B}_{p} \in\left(\mathcal{L}^{\infty}(\Omega)\right)^{3 \times 3} ; B_{i j} \text { piecewise linear, } \mathbf{B}_{p} \text { non negative definite }\right\}
\end{aligned}
$$

\subsubsection{Stating a minimization problem}

The following properties of the functional $\mathcal{F}$, demonstrated in [4] are the key of the extended identification procedure:

(i) $\mathcal{F}\left(\boldsymbol{\tau}, \mathbf{B}_{e}^{s}\right) \geq 0, \forall\left(\boldsymbol{\tau}, \mathbf{B}_{e}^{s}\right) \in \Sigma_{a d m} \times \xi_{e}$

(ii) $\mathcal{F}\left(\boldsymbol{\tau}, \mathbf{B}_{e}^{s}\right)=0 \Leftrightarrow(6)$ is true with $\boldsymbol{\sigma}=\boldsymbol{\tau}$ and $\mathbf{A}_{e}^{s}=\mathbf{B}_{e}^{s}$

(iii) The functional $\mathcal{F}$ is convex on $\Sigma_{a d m} \times \xi_{e}$

These properties also hold true for the functional $\mathcal{G}\left(\Delta \boldsymbol{\sigma}, \mathbf{B}_{e}^{t}\right)$ with $\left(\Delta \boldsymbol{\sigma}, \mathbf{B}_{e}^{t}\right) \in\left(\Delta \Sigma_{a d m}, \xi_{e}\right)$. They also apply to the plastic secant functional $\left(\mathcal{F}\left(\boldsymbol{\sigma}, \mathbf{B}_{p}^{s}\right)\right.$ with $\left.\left(\boldsymbol{\sigma}, \mathbf{B}_{p}^{s}\right) \in\left(\Sigma_{a d m}, \xi_{p}\right)\right)$ and to the plastic tangent functional $\left(\mathcal{G}\left(\Delta \boldsymbol{\sigma}, \mathbf{B}_{p}^{t}\right)\right.$ with $\left.\left(\Delta \boldsymbol{\sigma}, \mathbf{B}_{p}^{t}\right) \in\left(\Delta \Sigma_{a d m}, \xi_{p}\right)\right)$. For each secant or tangent behavior, the identification problem is thus equivalent to a minimization of the corresponding functional. Based on these properties, an adapted numerical strategy is developed in the next paragraph.

\subsection{Numerical implementation}

The first step of the numerical minimization of the functionals $\mathcal{F}$ and $\mathcal{G}$ is to build a finite element method allowing an appropriate description of displacement, stress, and material properties in the $\Omega$ domain. Using a finite element formulation, it becomes easy to compute the integrals defining the functionals $\mathcal{F}$ and $\mathcal{G}$. Equations (13) and (14) show that $\boldsymbol{\tau}$ and $\Delta \boldsymbol{\tau}$ do not depend on displacement and, consequently, three specific finite element formulations can be adopted for stress, displacement, and material properties fields. Using independent stress and displacement elements constitutes a particularity of this approach compared with the classical finite element methods applied to direct problems. 


\subsubsection{Finite Element Description}

\section{Stress interpolation}

The stress field solution has to satisfy the local equilibrium (eq. 5 or eq. 8). To enforce this constraint, a first method consists in using Lagrange multipliers. The two major drawbacks of this method both are : the size of the system largely increases while its conditioning deteriorates.

To decrease the number of Lagrange multipliers and to render the stress computation more straightforward, we choose a stress formulation based on naturally equilibrated Airy functions [10]. On each stress element, the Airy potential takes the polynomial form :

$$
\varphi(x, y)=\sum_{i=0}^{3} \sum_{j=0}^{3} a_{i j} x^{i} y^{j}
$$

In accordance with this choice, the 3 stress components are :

$$
\begin{aligned}
& \boldsymbol{\tau}_{x x}(x, y)=\varphi_{, y y}=\sum_{i=0}^{3} \sum_{j=2}^{3} j(j-1) a_{i j} x^{i} y^{j-2} \\
& \boldsymbol{\tau}_{y y}(x, y)=\varphi_{, x x}=\sum_{i=2}^{3} \sum_{j=0}^{3} i(i-1) a_{i j} x^{i-2} y^{j} \\
& \boldsymbol{\tau}_{x y}(x, y)=-\varphi_{, x y}=-\sum_{i=1}^{3} \sum_{j=1}^{3} i j a_{i j} x^{i-1} y^{j-1}
\end{aligned}
$$

\section{Displacement interpolation}

Once stress interpolation is chosen, interpolation of the displacements must be defined. We have chosen the $Q 2{ }_{9}$ element, constituted of 9 displacement nodes, and associated with a quadratic strain generating, via the constitutive equation, a quadratic stress $\mathbf{B} \varepsilon\left(\mathbf{u}^{*}\right)$ close to the stress $\boldsymbol{\tau}$ given by the Airy potentials. This element constitutes a good compromise between complexity of formulation and richness of strain description.

\section{Material coefficients interpolation}

Finally, the material properties are considered to be constant within each element. Nevertheless, we have the possibility to define sets of elements with equal material properties. This possibility is particularly interesting when several extended homogeneous material zones coexists in $\Omega$.

Naturally, the stress, the displacement, and the material elements have the same geometrical support. For the moment, our meshing procedure is dealing with simple geometries, where elements are rectangular, but an extension to more general meshes is envisaged.

The functionals $\mathcal{F}$ and $\mathcal{G}$ can then be computed and minimized using these finite element descriptions. The convexity of these functionals makes the use of a relaxation method particularly relevant. This method consists in minimizing successively the functionals over their first and second variables respectively. It requires measured displacement fields, measured reactions, and an a priori information relative to the stress-free boundaries available. Naturally, stress minimization and material minimization are performed alternatively until convergence occurs. Convergence is checked by comparing both the material parameters and the stress between the two last iterations. The convex properties of the functionals ensure the existence of a solution for each of the two minimization problems. 


\subsubsection{First minimization : computation of the stress field}

For both elastic and plastic loadings, the same method is used to compute stress or stress increment, in order to simplify the stress computation procedure (cf. "stress minimization" in Figure $1)$.

In all situations, minimizing $\mathcal{F}$ or $\mathcal{G}$ over the stress is equivalent to solving the $K U=F$ system, where $U$ is the nodal Airy potential vector containing the values $\left(\varphi, \frac{\partial \varphi}{\partial x}, \frac{\partial \varphi}{\partial y}, \frac{\partial^{2} \varphi}{\partial x \partial y}\right)$ at each node, $K$ is the Hessian of $\mathcal{F}$ or $\mathcal{G}$ over $U$ and $F$ is the gradient of $\mathcal{F}$ or $\mathcal{G}$ over $U$ computed at the point $U=0$. The constraints corresponding to observations at the boundary (eq. 7 or 10) are superimposed to this system using Lagrange multipliers.

For fine meshes, the $K U=F$ linear system is large and often badly conditioned (due to the Lagrange multipliers used to impose a global equilibrium), which justifies the use of a conjugate gradient algorithm.

\subsubsection{Second minimization : computation of the material properties distributions}

In our strategy, the elastic properties are determined once and for all during the first loading steps, then the plastic parameters are computed considering the subsequent loading steps. The next two paragraphs focus on the computation of the elastic and plastic material coefficients.

\section{Elastic material properties computation}

At the most, three local material coefficients can be determined. We choose to identify cubic or isotropic elastic models here. The elastic tensor $\mathbf{B}_{e}$ can be computed indiscriminately by the standard or the incremental formulation. The minimization of $\mathcal{F}$ or $\mathcal{G}$ over $\mathbf{B}_{e}$ is explicit and leads directly to the two or three elastic coefficients of the chosen model (cf. Figure 1). The following description only deals with the "standard formulation", but can be directly adapted to the incremental one.

The minimization algorithm is presented in Figure 1. It requires an initial value $\mathbf{B}_{0}$ of the elastic matrix. As there is no influence of the initial guess on the obtained solution, due to the convex properties of $\mathcal{F}, \mathbf{B}_{0}$ is chosen arbitrarily. Naturally, bad initial guesses may extend the computational time.

The step called "elastic material characterization" corresponds to the local estimation of the elastic matrix $\mathbf{B}_{e}$. Note that two situations may occur in this latter step, depending on the mean equivalent strain $\|\varepsilon\|$ on the considered element :

- if $\|\varepsilon\|$ is too small compared with the maximal equivalent strain $\varepsilon_{\max }$ on the whole $\Omega$ domain (i.e. $\frac{\|\boldsymbol{\varepsilon}\|}{\varepsilon_{\max }}<\delta_{\epsilon}$ where $\delta_{\epsilon}$ is a threshold criterion on the strain), we consider that the deformation energy is too small to allow a robust identification of the material properties. Then, a substitution procedure is applied where the elastic matrix of the considered element is replaced by the homogenized $\mathbf{B}_{e}$ on a chosen subdomain of $\Omega$. It is important to note that even if the elastic properties of the substituted zones do not perfectly match the real ones, the corresponding error does not affect the stress computation because these zones are almost unstrained, and their weight in the overall deformation energy is therefore very small.

- If the local deformation is sufficient $\left(\frac{\|\boldsymbol{\varepsilon}\|}{\boldsymbol{\varepsilon}_{\max }} \geq \delta_{\epsilon}\right)$ then the elastic matrix $\mathbf{B}_{e}$ is computed by minimizing $\mathcal{F}\left(\boldsymbol{\tau}, \mathbf{B}_{e}\right)$ with respect to $\mathbf{B}_{e}$. 


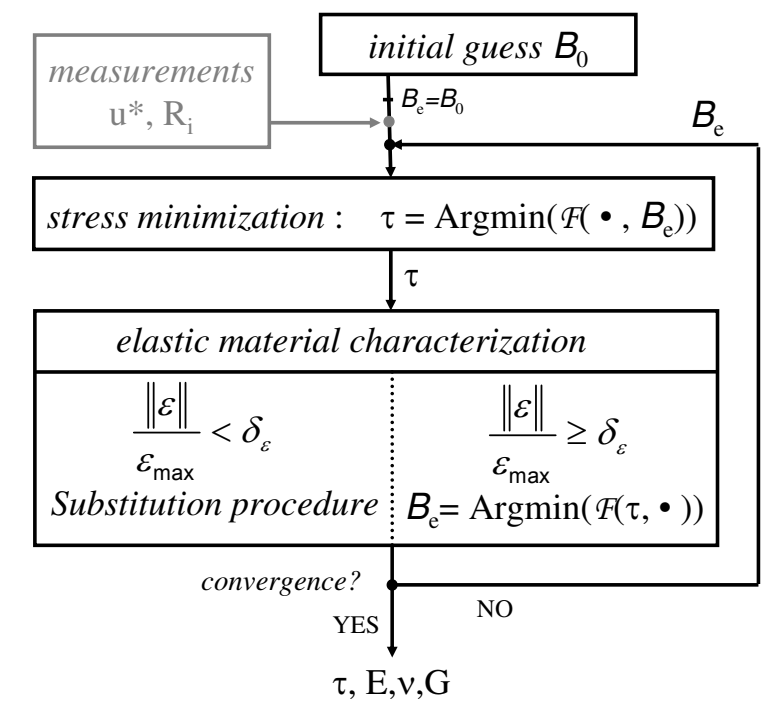

Figure 1: Algorithm used to compute the elastic properties

\section{Plastic material properties computation}

Concerning anelastic behavior, we restrict our attention to an elastoplastic model with isotropic kinematic hardening associated with a von Mises criterion. Two plastic parameters are identified locally: the kinematic hardening modulus $k$ and the yield stress $\sigma_{0}$.

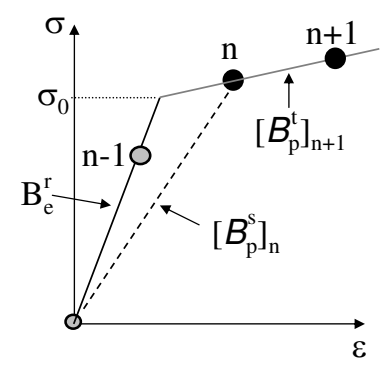

Figure 2: Simple diagram $(\boldsymbol{\sigma}, \boldsymbol{\varepsilon})$ corresponding to the elastoplastic model.

Denoting $\Delta \gamma$ the plastic multiplier increment, we express the elastoplastic secant tensor $\mathbf{A}_{p}^{s}$ and the tangent tensor $\mathbf{A}_{p}^{t}$ at the step $n$ according to [11]:

$$
\begin{aligned}
& {\left[\mathbf{A}_{p}^{s}\right]_{n}=\left[\mathbf{A}_{e}^{-1}-\frac{\Delta \gamma_{n}\left(\sigma_{0}\right)}{1+\frac{2}{3} k \Delta \gamma_{n}\left(\sigma_{0}\right)} \mathbf{P}\right]^{-1}} \\
& {\left[\mathbf{A}_{p}^{t}\right]_{n}=\left[\mathbf{A}_{p}^{s}\right]_{n}-\frac{N_{n} \otimes N_{n}}{1+\beta_{n}}}
\end{aligned}
$$

where $N_{n}$ is a vector linked to the normal to the load surface, $\beta_{n}$ is a scalar characterizing the hardening, and $\mathbf{P}$ is a mapping matrix. Their definition is given in the following equations: 


$$
\begin{aligned}
\beta_{n} & =\frac{2}{3} k\left(1+\frac{2}{3} \Delta \gamma k\right) \frac{\xi_{n}^{T} \mathbf{P} \xi_{n}}{\xi_{n}^{T} \mathbf{P}\left[\mathbf{A}_{p}^{s}\right]_{n} \mathbf{P} \xi_{n}} \\
N_{n} & =\frac{\left[\mathbf{A}_{p}^{s}\right]_{n} \mathbf{P} \xi_{n}}{\sqrt{\xi_{n}^{T} \mathbf{P}\left[\mathbf{A}_{p}^{s}\right]_{n} \mathbf{P} \xi_{n}}} \\
\Delta \gamma & =\frac{3}{2 k}\left(\sqrt{\frac{3}{2}} \frac{\alpha}{\sigma_{0}}-1\right)
\end{aligned}
$$

where

$$
\begin{aligned}
\xi_{n} & =\frac{1}{1+\frac{2}{3} \Delta \gamma k}\left(\boldsymbol{\sigma}_{n}-\mathbf{X}_{n-1}\right) \\
\mathbf{X}_{n} & =\frac{1}{1+\frac{2}{3} \Delta \gamma k}\left(\mathbf{X}_{n-1}+\frac{2}{3} \Delta \gamma k \boldsymbol{\sigma}_{n}\right) \\
\alpha^{2} & =\left(\boldsymbol{\sigma}_{n}-\mathbf{X}_{n-1}\right)^{T} \mathbf{P}\left(\boldsymbol{\sigma}_{n}-\mathbf{X}_{n-1}\right) \\
\mathbf{P} & =\frac{1}{3}\left[\begin{array}{ccc}
2 & -1 & 0 \\
-1 & 2 & 0 \\
0 & 0 & 6
\end{array}\right]
\end{aligned}
$$

For the first plastic step (i.e. step $n$ in Figure 2 ) it is common to write a secant formulation of the plastic problem.

Remark 1 The three equations obtained by computing the gradient of $\mathcal{F}$ over $\mathbf{A}_{p}^{s}$ are dependent and do not allow to identify both $k$ and $\sigma_{0}$ on a single loading step.

Consequently, an iterative algorithm involving two successive plastic loading steps denoted $n$ and $n+1$ have been carried out. Since all the minimization steps are now detailed, the next paragraph will focus on the description of the specific elastoplastic algorithm.

\subsubsection{Resolution algorithm}

As mentioned previously, the reference elastic tensor, noted $\mathbf{B}_{e}^{r}$, is obtained on the first loading step. Then, on each following loading step, noted $n$, a procedure named "comparison procedure", presented in Figure 3, aims at comparing a local tangent elastic tensor $\left[\mathbf{B}_{e}^{t}\right]_{n}$ with the reference elastic tensor $\mathbf{B}_{e}^{r}$. $\left[\mathbf{B}_{e}^{t}\right]_{n}$ is obtained by minimizing $\mathcal{G}\left(\boldsymbol{\tau}_{n},\left[\mathbf{B}_{e}^{t}\right]_{n}\right)$ on the load increment $n$ using the following input data : the reaction increment $\left[\Delta R_{i}\right]_{n}$ and the incremental displacement field $\left[\Delta \mathbf{u}^{*}\right]_{n}$. This procedure is only applied to the most strained elements for which $\frac{\|\Delta \varepsilon\|}{\Delta \varepsilon_{\max }} \geq \delta_{\epsilon}$. For the less strained elements, it is assumed that no local plasticity occurs and $\left[\mathbf{B}_{e}^{t}\right]_{n}$ is equal to the reference elastic tensor $\mathbf{B}_{e}^{r}$.

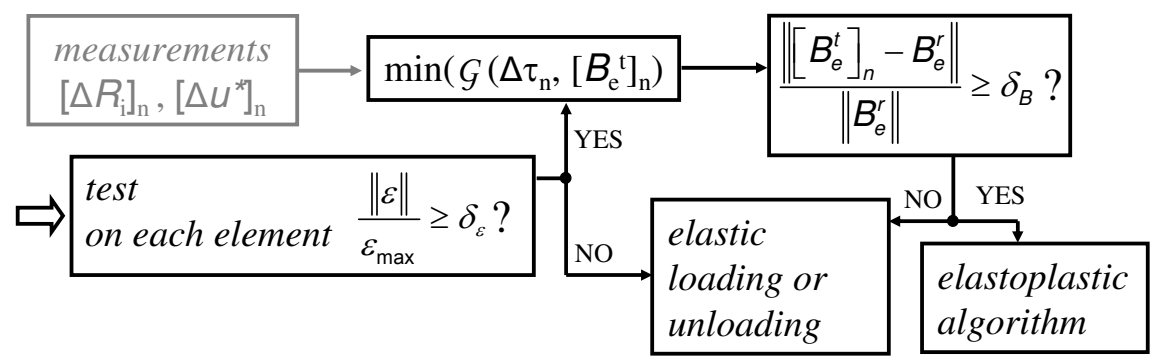

Figure 3: Initialization of the plastic computation 
The elements where the comparison procedure detects any changes in the local stiffness tensor (i.e. $\frac{\left\|\left[\mathbf{B}_{e}^{t}\right]_{n}-\mathbf{B}_{e}^{r}\right\|}{\left\|\mathbf{B}_{e}^{r}\right\|} \geq \delta_{B}$ ) are assumed to be plastic, and an elastoplastic algorithm (cf. Figure 4) allows to identify separately the two plastic parameters $k$ and $\sigma_{0}$. Moreover, this procedure allows to discriminate the elastically unloaded zones of the specimen from the plastically loaded ones that can be observed under certain conditions (Lüders bands, necking, ...).

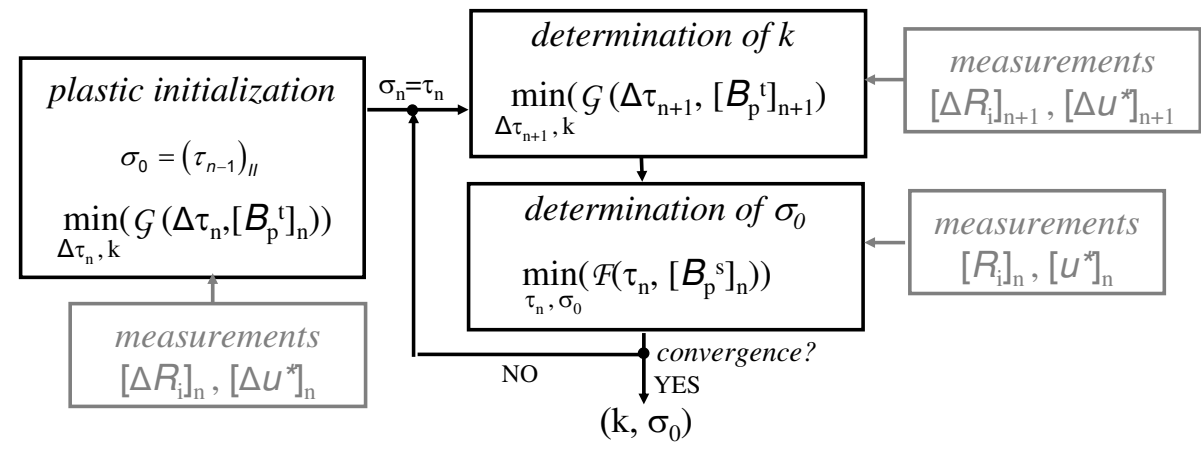

Figure 4: elastoplastic algorithm

In the first step of the elastoplastic algorithm presented in Figure 4, named "plastic initialization", we assume that $\sigma_{0}=\left(\boldsymbol{\tau}_{n-1}\right)_{I I}$ where $(\cdot)_{I I}$ stands for the von Mises norm. Thus, we compute an approximative hardening modulus $k$ by minimizing $\mathcal{G}\left(\Delta \boldsymbol{\tau}_{n},\left[\mathbf{B}_{p}^{t}\right]_{n}\right)$, where $\left[\mathbf{B}_{p}^{t}\right]_{n}$ stands for the plastic tangent modulus at the step $n$, depending on $k$. This minimization requires measures of the reaction increment $\left[\Delta R_{i}\right]_{n}$ and the incremental displacement field $\left[\Delta \mathbf{u}^{*}\right]_{n}$. The goal of this initialization procedure is to obtain an approximation of the backstress $\mathbf{X}_{n}$ at step $n$ needed in the first step of the iterative algorithm named "determination of $k$ ", the second step being "determination of $\sigma_{0}{ }^{\prime \prime}$.

- "determination of $k$ " : we minimize $\mathcal{G}\left(\Delta \boldsymbol{\tau}_{n+1},\left[\mathbf{B}_{p}^{t}\right]_{n+1}\right)$, where $\left[\mathbf{B}_{p}^{t}\right]_{n+1}$ stands for the plastic tangent modulus at the step $n+1$, depending on $k$. This minimization requires some measurements inputs (the reaction increment $\left[\Delta R_{i}\right]_{n+1}$, the incremental displacement field $\left.\left[\Delta \mathbf{u}^{*}\right]_{n+1}\right)$, and the backstress $\mathbf{X}_{n}$ previously computed.

- "determination of $\sigma_{0}$ " : this consists in minimizing $\mathcal{F}\left(\boldsymbol{\tau}_{n},\left[\mathbf{B}_{p}^{s}\right]_{n}\right)$, where $\left[\mathbf{B}_{p}^{s}\right]_{n}$ stands for the plastic secant modulus at the step $n$, depending on $\sigma_{0}$. This minimization also requires some measurements inputs (the reaction $\left[R_{i}\right]_{n}$, the displacement field $\left[\mathbf{u}^{*}\right]_{n}$ ), and the previously estimated value of $k$.

\section{Applications}

In this part, both numerical and experimental results are presented. In a first instance, the method is tested on the basis on simulated data given by the resolution of a direct problem. Note that the meshes used for direct computation and identification are always different, the finest being always the one used for the direct finite element computation, in order to minimize errors on the direct problem over errors due to the identification method.

In order to characterize the spatial resolution of the method, we decided to work on a composite specimen made of two different materials. As the solution to inverse problems are known to be very sensitive to small perturbations on the input data [12], we illustrate the robustness of this method by studying its sensitivity study to a superimposed noise on this composite specimen.

Finally, results obtained experimentally on a similar configuration are presented. 


\subsection{Numerical identification on a bi-material composed sample}

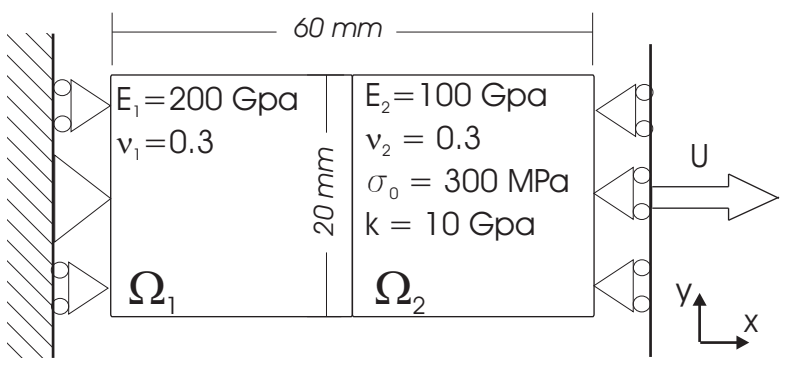

(a) numerical simulation

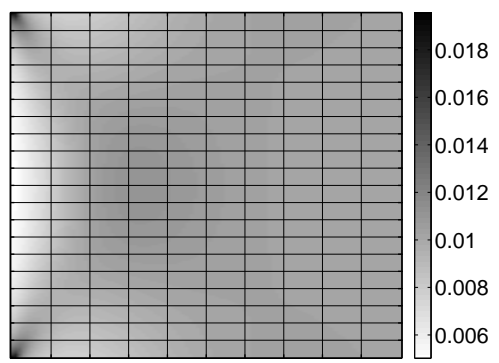

(b) $\left\|\epsilon_{p}\right\|$ on $\Omega_{2}$

Figure 5: Description of the test (a) and equivalent plastic strain plotted with the mesh of the identification method (b).

A tensile test on a bi-material sample is simulated for the loading conditions shown in Figure 5a. This simulation by a 2D F.E.M. uses $90 \times 60$ Q1 elements. The loaded barrel is made of two material zones. The $\Omega_{1}$ zone is purely elastic and the $\Omega_{2}$ zone, less stiff, is elastoplastic with linear kinematic hardening. This test is highly penalizing due to strong strain gradients in the vicinity of the interface. The objective here is to test the performance of our method in presence of material heterogeneities. Identification has been performed for an ideal configuration, where the boundary between material domains $\Omega_{1}$ and $\Omega_{2}$ is coincident with the meshing of the identification procedure.

\subsubsection{Noise sensitivity in elasticity}

The robustness of the method is, in a first step, tested for the elastic identification. The mesh of the identification method uses $20 \times 20$ material zones corresponding to $41 \times 41$ displacement nodes. A Gaussian white noise is added to the assumed-to-be-exact displacements obtained by the direct F.E.M. computation. Denoting $\left(\varepsilon_{1}, \varepsilon_{2}, \varepsilon_{3}\right)=\left(\varepsilon_{x x}, \varepsilon_{y y}, \varepsilon_{x y}\right)$, we define a noise ratio $r_{n o i s e}$ on the strain:

$$
r_{\text {noise }}=\max _{i=1 \cdots 3}\left(\frac{\sup _{\Omega}\left|\varepsilon_{i}\left(\mathbf{u}_{\text {noisy }}^{*}\right)-\boldsymbol{\varepsilon}_{i}\left(\mathbf{u}_{r e f}^{*}\right)\right|}{\sup _{\Omega}\left|\varepsilon_{i}\left(\mathbf{u}_{r e f}^{*}\right)\right|}\right)
$$

where $\varepsilon_{i}$ stands for the $i^{\text {th }}$ component of the strain. The strain component may be derived either from the displacement $\mathbf{u}_{r e f}^{*}$ issued by direct F.E.M. computation, or by noisy displacement $\mathbf{u}_{n o i s y}^{*}$. The sensivity study has been performed for a $r_{n o i s e}$ ratio varying from 0 to $50 \%$. The maximum ratio $r_{\text {noise }}$ is associated with a peak-to-peak noise amplitude of $4 \times 10^{-4}$ on strain measurement, which is two times higher than the experimental resolution on strain measurement [1]. The evolutions of the absolute error on the identification of the parameters $E$ et $\nu$ using an isotropic elastic model are progressive ( $c f$. Figure 6). The maximal error is worth $20 \mathrm{GPa}$ for Young's modulus, which gives a relative error inferior to $20 \%$. For the Poisson ratio, the maximal error is worth 0,07 i.e. a $24 \%$ relative error. 


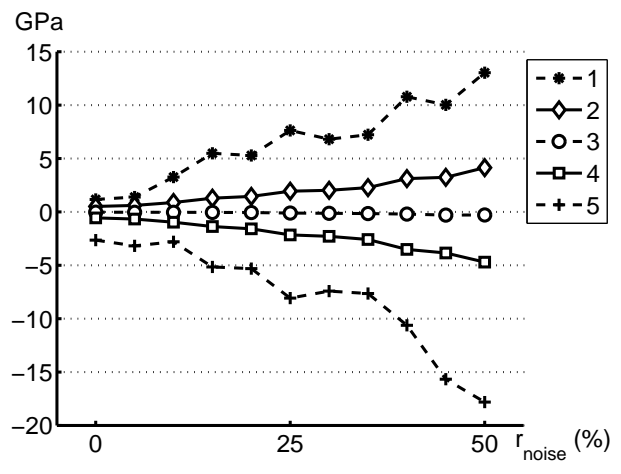

(a) Young modulus $E$

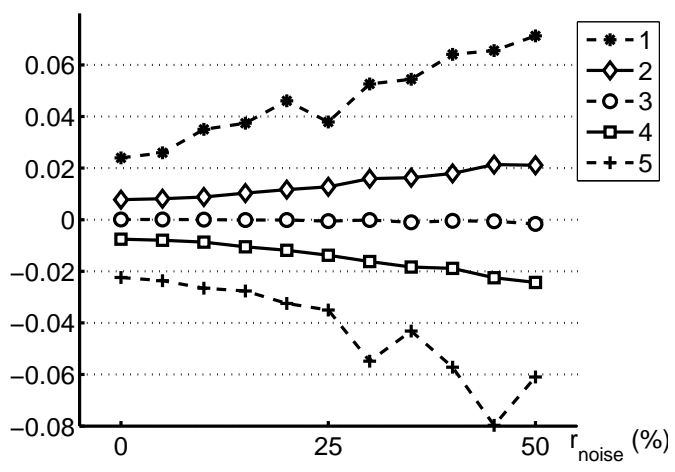

(b) Poisson ratio $\nu$

Figure 6: Absolute errors associated with the local determination of material parameters: maximal gap (1), mean error + standard deviation (2), mean error (3) , mean error - standard deviation $(4)$, minimal gap (5)

Figure 7 represents a 3D-surface of the identified Young modulus for the maximal noise ratio $r_{n o i s e}=50 \%$, it is noticeable that the boundary is perfectly located and this step-like surface reflects perfectly the real hehavior of the harrel even if small nertuhations can be observed.

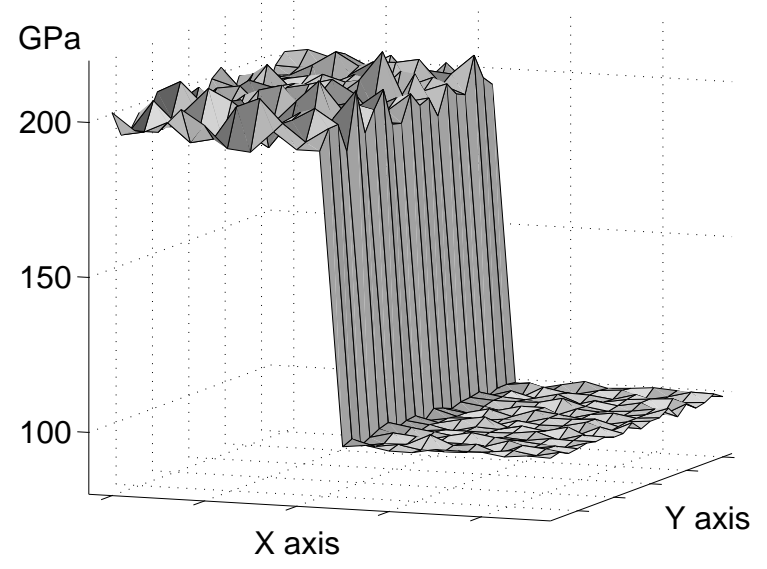

Figure 7: Young modulus identified from noisy data with $r_{n o i s e}=50 \%$

\subsubsection{Plastic parameter distributions}

First, we present some results obtained from displacements directly given by simulation without superimposing any noise. The material parameters are, again, identified on $20 \times 20$ material zones. The barrel does not plastify uniformly over $\Omega$ : the $\Omega_{1}$ domain remains elastic and the plasticity heterogeneously develops over $\Omega_{2}$ (cf. Figure $5 \mathrm{~b}$ ). The values of criteria $\delta_{\epsilon}$ and $\delta_{B}$ are $5 \%$ for both. Using these criteria, plasticity is only identified on the elements located within the $\Omega_{2}$ domain. Consequently, and as the interface between the material zones is coincident with the meshing of the identification method, the interface between $\Omega_{1}$ and $\Omega_{2}$ is perfectly attained.

Figure 8 illustrates $\sigma_{x x}$ tensile stress distributions at the end of the first plastic step. The stress presented in Figure 8a is obtained by solving the direct problem, whereas the one given in Figure $8 \mathrm{~b}$ has been identified by the inverse method. We notice a good similarity between the distributions, and the orders of magnitude for this stress component. In particular, the stress gradients are correctly transcribed in the neighborhood of the interface. 


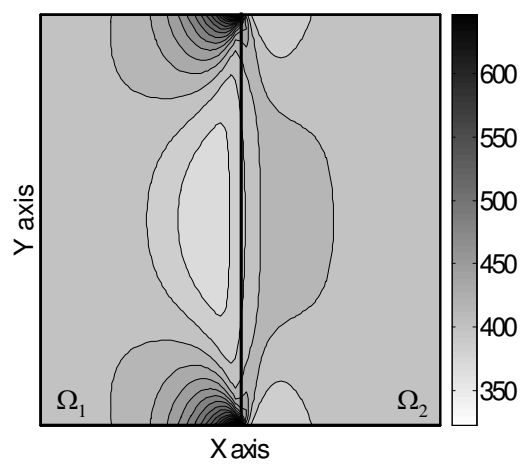

(a) reference stress $(\mathrm{MPa})$

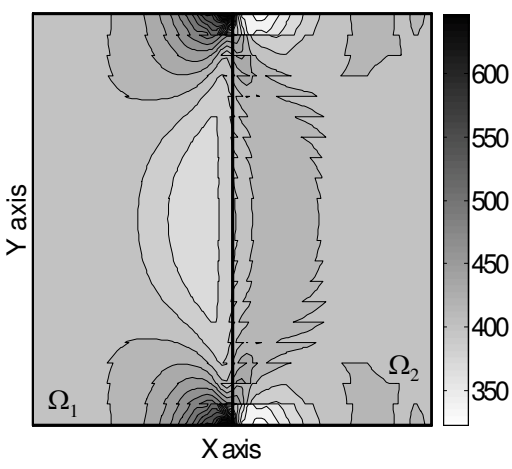

(b) identified stress $(\mathrm{MPa})$

Figure 8: Comparison for the first plastic step ( $\operatorname{step} n=2$ ) between the tensile stress component given by the direct computation and the same component given by the identification method.

Figure 9 illustrates the distribution of the identified plastic parameters. We clearly observe that the identification error on the two parameters is not randomly distributed. It is more important in the elements where plastic strain is small and where the strain gradients are important.

On the hardening modulus $k$, the maximum error observed is $23 \%$, corresponding to $2.3 \mathrm{GPa}$ that is comparable with the error obtained on Young's modulus identification when no noise is added on the data ( $c f$. Figure 6a). The maximum error on $\sigma_{0}$ is $14 \%$ ( $c f$. Figure $9 \mathrm{~b}$ ).

The presence of strain gradients is an important source of errors of identification. As the mesh used for identification is coarser than the one used for the direct computation, it does not render the stress distribution properly in the vicinity of the interface.

Another error source may result from direct computation, which may be less accurate in the case of non-linear problems.

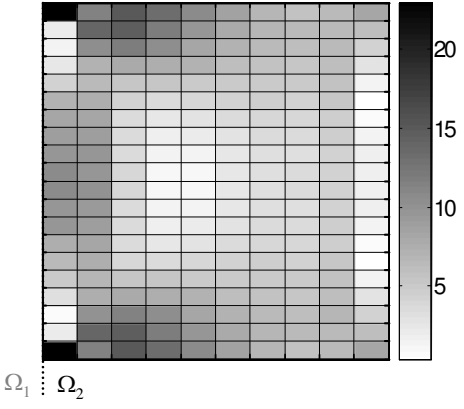

(a) error on $k$ in $\%$

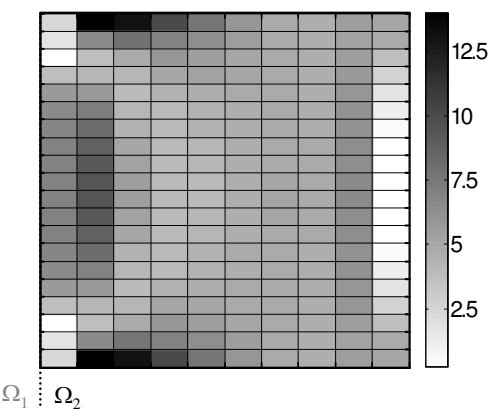

(b) error on $\sigma_{0}$ in $\%$

Figure 9: Relative errors in determining the plastic parameters plotted on $\Omega_{2}$ only (elements in the $\Omega_{1}$ domain are not represented because they are not identified as plastic). 


\subsubsection{Noise sensitivity in plasticity}

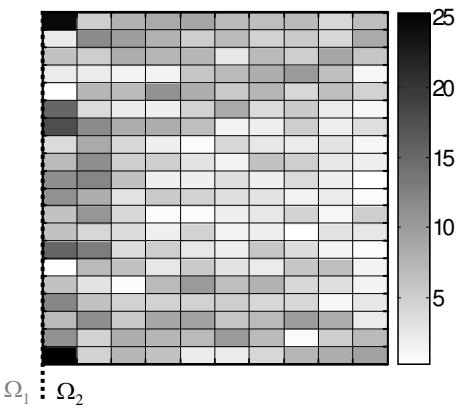

(a) error on $k$ in $\%$

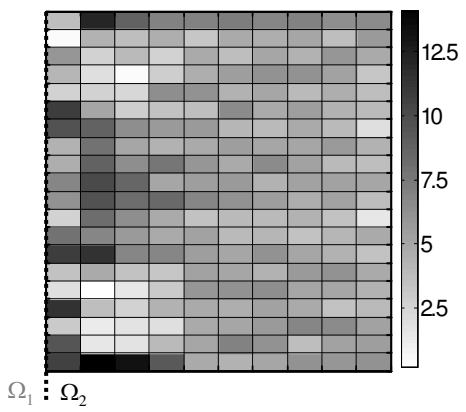

(b) error on $\sigma_{0}$ in $\%$

Figure 10: Relative errors in determining the plastic parameters identified from noisy data $\left(r_{\text {noise }}=25 \%\right)$

A noise sensitivity study similar to the one performed in the elastic case has been performed for the simulation already presented in Figure 5. On each loading step, a Gaussian white noise is added to the simulated displacement increment. To facilitate a comparison with the elastic study, we adopt the following definition of the noise ratio $r_{\text {noise }}$ :

$$
r_{\text {noise }}=\max _{i=1 \cdots 3}\left(\frac{\sup _{\Omega}\left|\varepsilon_{i}\left(\Delta \mathbf{u}_{\text {noisy }}^{*}\right)-\varepsilon_{i}\left(\Delta \mathbf{u}_{r e f}^{*}\right)\right|}{\sup _{\Omega}\left|\varepsilon_{i}\left(\Delta \mathbf{u}_{r e f}^{*}\right)\right|}\right) .
$$

On each loading step (elastic step, and plastic $n$ and $n+1$ steps) we add a displacement field related to similar $r_{n o i s e}$ ratio ranging from 0 to $50 \%$. Thus, the plastic identification starts from an elastic set of parameters identified with noisy data. The corresponding error on the elastic parameters used as inputs in the plastic identification is given in Figure 6. In Figure 10, we observe that the error distribution on the identified parameters shows a similar pattern as the one corresponding to "perfect" data. To isolate the influence of superimposed noise from this systematic bias, we have chosen to represent ( $c f$. Figure 11) the differences between the values identified using noisy and "perfect" data. The error associated with superimposed noise is randomly distributed (its mean value remains close to zero) and the standard deviation increases linearly with noise amplitude, as observed in the elastic case.

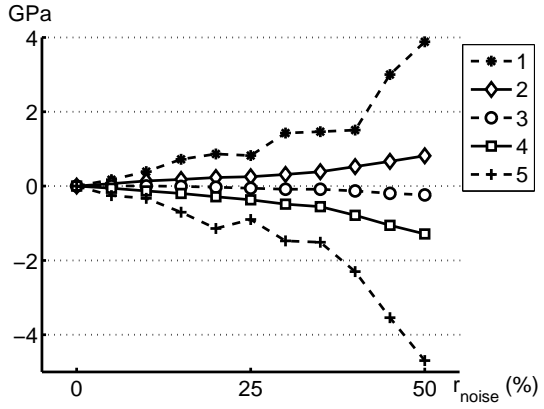

(a) hardening modulus $k$

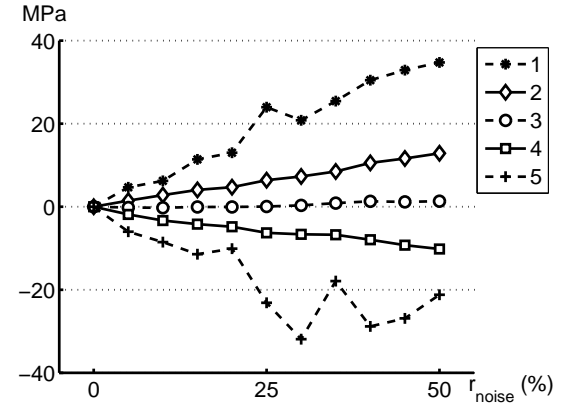

(b) yield stress $\sigma_{0}$

Figure 11: Errors relative to the local determination of material parameters: maximal gap (1), mean error + standard deviation (2), mean error (3) , mean error - standard deviation (4), minimal gap (5). 


\subsection{Experimental identification}

\subsubsection{Description of the specimen and its material properties}

The experiment presented in this part was chosen to reproduce the previous numerical simulation. The sample is a thin flat dog-bone specimen machined in a $2.5 \mathrm{~mm}$ thick plate of DP60 steel. The bimaterial behavior is artificially recreated by a thickness variation : the thickness of the zone where $x \in[-28 \mathrm{~mm}, 0]$ was symmetrically reduced to $0.8 \mathrm{~mm}$ (cf. Figure 12).

In the identification procedure, thickness is supposed to be constant on the whole specimen (i.e. $0.8 \mathrm{~mm}$ ). Thus we expect to identify the reference properties in the thin zone, and properties of a stiffer material in the thicker zone.

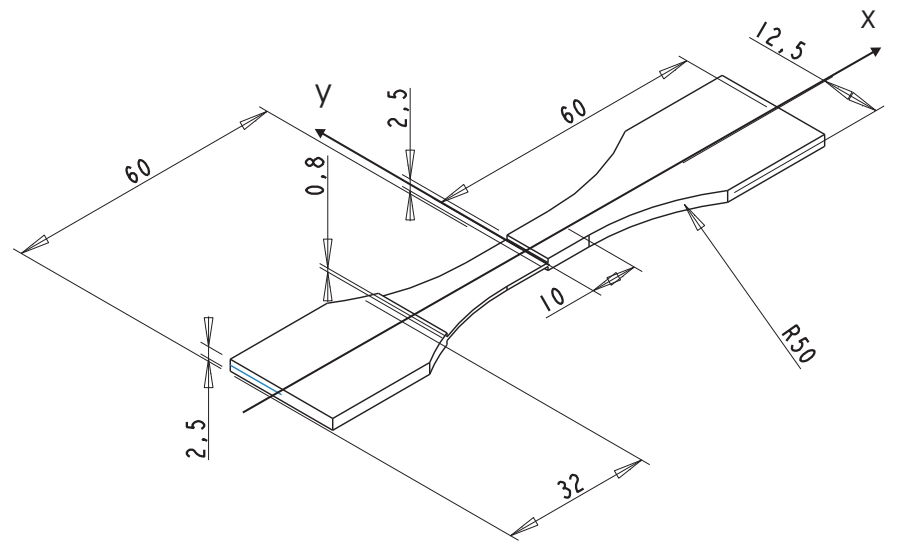

Figure 12: Shape of the specimen (dimensions in $\mathrm{mm}$ )

The $\Omega$ identified zone consists of a square $(20 \mathrm{~mm} \times 20 \mathrm{~mm})$ centered on the specimen meshed by $10 \times 10$ square elements. We assume the existence of a plane stress state, neglecting the outof-plane effects induced by the thickness discontinuity. Any triaxial effects in the vicinity of the discontinuity would naturally affect the identified parameters.

A preliminary tensile test on a specimen of the same steel, the same geometry but with a constant thickness of $2.5 \mathrm{~mm}$ has been performed to obtain the reference elastoplastic properties of the material. The stress-strain diagram corresponding to this test is presented in Figure 13. The first linear part of the diagram allows to identify the elastic parameters $(E=207 \mathrm{GPa}$, $\nu=0.27)$. From this diagram, we obtain the following parameters of the elastoplastic model : $k=5 \mathrm{GPa}, \sigma_{0}=370 \mathrm{MPa}$. On this material, the first stages off the hardening are rather nonlinear, and the values of the plastic parameters associated with a linear kinematic hardening will be very dependent with respect to the strain levels for which the identification is performed. For that reason, we have located, in Figure 13, the three strain levels used to determine the reference plastic parameters.

The local identification method is carried out on the bimaterial sample for three strain levels. These levels are obtained by matching the maximal local strain on the identified domain with the isolated strain level on the diagram of the homogeneous tensile test. 


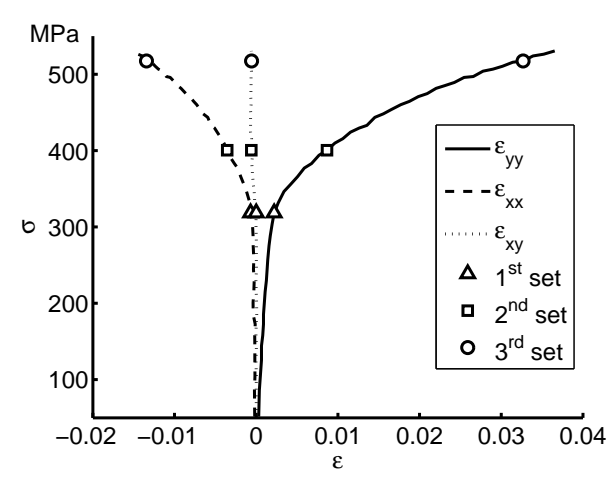

Figure 13: Stress-strain diagram of a simple tensile test on DP60 steel

The displacement measurement given by D.I.C. is filtered using calibrated splines and its efficiency is presented in Figure 14. It illustrates the tensile component of the displacement $U_{x}$ associated with the first strain level.

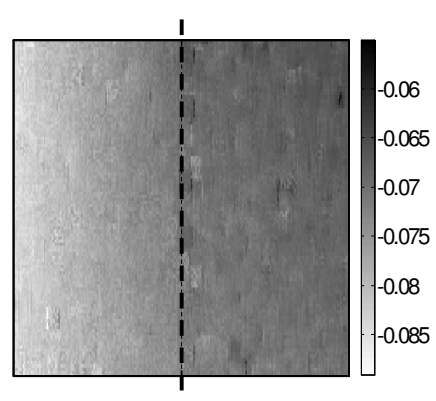

(a) $U_{x}$ given by D.I.C

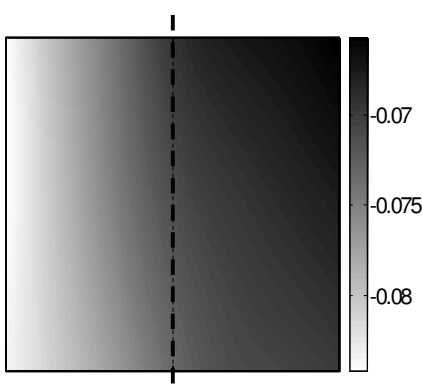

(b) $U_{x}$ filtered

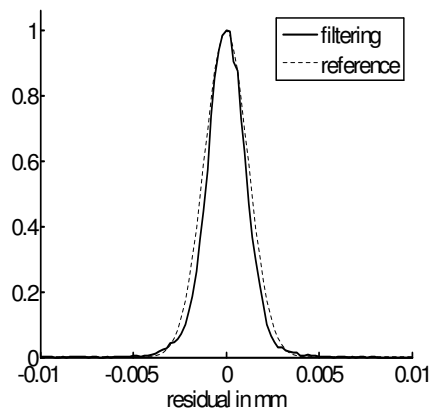

(c) filtering residual

Figure 14: Filtering of the $U_{x}$ displacement field obtained by D.I.C.

A "filtering" histogram plotted in Figure 14c is related to the filtering residual defined as the difference between the measured and filtered displacements, computed for the first strain level. A "reference" Gaussian histogram is plotted with a scattered line, it is obtained from the mean error and standard deviation of the "filtering" histogram. The two curves almost coincide one with another, moreover the "filtering" histogram is centered around zero, which corresponds to a negligible systematic filtering error. The standard deviation is worth $1.2 \times 10^{-3} \mathrm{~mm}$, i.e. $6 \%$ of the displacement amplitude. The signal/noise ratio increases for the following strain levels. This strongly suggests that identification will be more accurate for the plastic parameters.

\subsection{2 identification results}

The identified elastic parameter distribution is presented in Figure 15. The interface between the thick and the thin part is plotted with a vertical scattered line dividing the identification domain $\Omega$ into two equal subdomains. The mesh of the identification procedure was adjusted to match the interface perfectly. Let $\Omega_{1}$ be the left part of $\Omega$ corresponding to the thin part of the specimen, and $\Omega_{2}$ the right, thick part of $\Omega$.

Evidently $\Omega_{2}$ is weakly deformed, thus the values identified on the four last columns of the mesh are substituted, following the procedure presented in section 2.2.3. We choose to substitute these values by the homogeneous elastic coefficients in $\Omega_{2}$. 


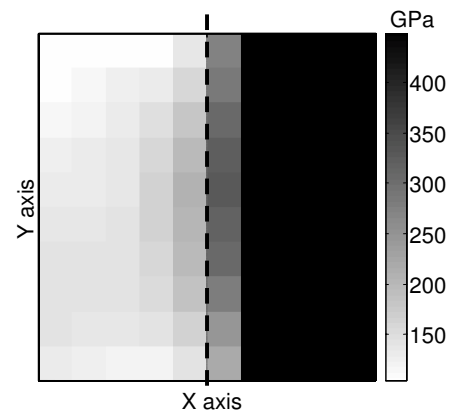

(a) Young modulus $E$

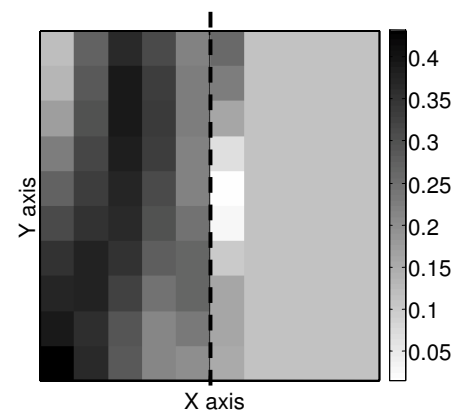

(b) poisson ratio $\nu$

Figure 15: Identified elastic properties

Concerning Young's modulus, the values obtained on $\Omega_{1}$ are centered around $150 \mathrm{GPa}$, which is lower than the reference value obtained in the homogeneous tensile test. This is not surprising given that, for this first step which we assume to be elastic, the average deformation on $\Omega_{1}$ is nearly two times the one used to obtain the reference elastic parameters. In this zone, the dispersion is small, and the modulus increases near the interface. This first identified column in $\Omega_{2}$ has been identified locally. The mean value of this column is worth $290 \mathrm{GPa}$, which is between values expected for $\Omega_{1}$ (206 GPa) and values expected for $\Omega_{2}$ (643 GPa, depending on variations in thickness of our specimen). The mean value of $E$ identified for $\Omega_{2}$ is nearly $450 \mathrm{GPa}$, which is coherent with the thickness ratio between the two subdomains.

Concerning the Poisson ratio $\nu$, the results are rather scattered. Nevertheless the average identified value on the $\Omega_{1}$ domain (0.3) is consistent with the reference value (0.27). On the four last columns of $\Omega_{2}$, the substitution value is worth 0.11 , but this error is due to contraction strain component, which is rather low on the stiff part of the specimen.

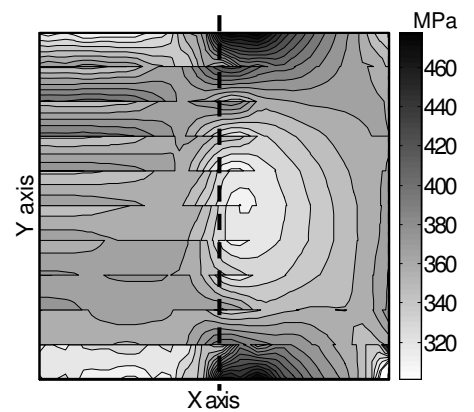

(a) tensile stress $\boldsymbol{\sigma}_{x x}$

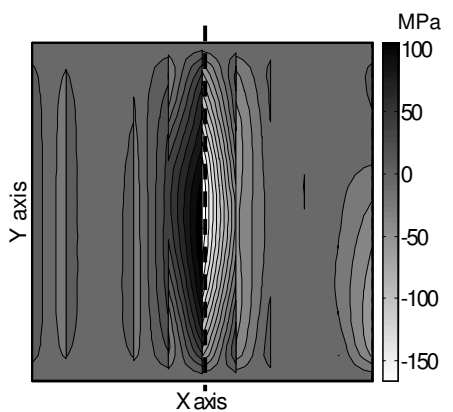

(b) contraction stress $\boldsymbol{\sigma}_{y y}$

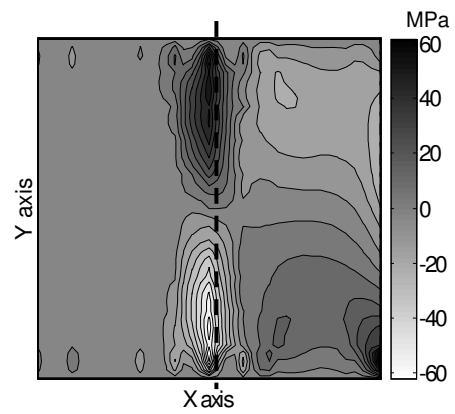

(c) shear stress $\boldsymbol{\sigma}_{x y}$

Figure 16: Identified components of the total stress $\boldsymbol{\sigma}_{n}$ at the second loading step

The stress distributions presented in Figure 16 are obtained for the plastic step $n=2$, and correspond to the second strain level. The maximum tensile strain on the fields computed for this step is situated around $2 \%$. The stress fields obtained in this experimental study are very similar to those given by numerical simulation. In particular, the contraction $\boldsymbol{\sigma}_{y y}$ and shear stress $\boldsymbol{\sigma}_{x y}$ are perfectly located on the interface, and the profiles of the two stress components are realistic. The high concentrations of the tensile components $\boldsymbol{\sigma}_{x x}$ are located on the two ends of the interface, and similar to the field identified by simulation (cf. Figure 8).

Owing to the particular interpolation of the stresses, $\boldsymbol{\sigma}_{x x}$ and $\boldsymbol{\sigma}_{y y}$ are discontinuous with respect to the $y$ and $x$ axes, respectively. The main discontinuities appear when the variation in material properties from one element to another is high. 


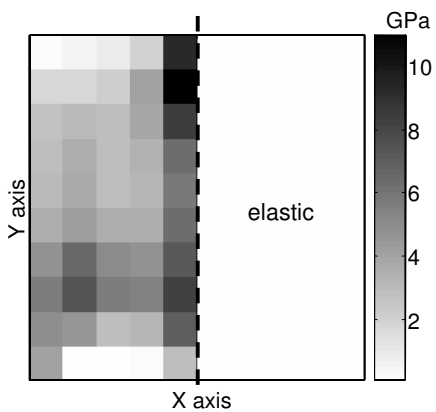

(a) hardening modulus $k$

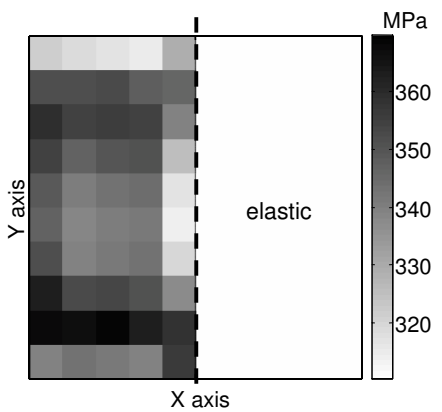

(b) yield stress $\sigma_{0}$

Figure 17: Identified plastic properties

The plastic properties distributions are presented in Figure 17. Here, the shape of the of the interface between the plastic zone and the elastic one is consistent with the mesh used in the identification procedure, and the interface is perfectly localized. Identification of the yield stress $\sigma_{0}$ gives very satisfying results centered around a mean value of $340 \mathrm{MPa}$ which is $8 \%$ inferior to the reference one. The field obtained is nearly symmetric with respect to the $(O x)$ axis, due to the symmetry of the stress field.

This identified hardening modulus $k$ is higher than the reference value near the interface, where its mean value is worth $8 \mathrm{GPa}$. This may be due to the spatial filtering which tends to homogenize the identified properties. The few elements where the obtained $k$ is very low are the elements where the stress is very close to the yield stress ( $c f$. Figure 16). In this case the plastic strain energy is too weak to allow an accurate identification of the plastic properties. Everywhere else, the modulus $k$ is rather homogeneous and its mean value is worth $4 \mathrm{GPa}$ corresponding to the reference one.

Already, the results of this identification are encouraging. In this experiment where strain fields are heterogeneous and where plasticity heterogeneously develops, the contrast on the material properties between two zones of different apparent stiffness has been well identified. Moreover, the quality of the identification method is enforced by the agreement between local properties identified from the bi-thickness sample and homogeneous properties obtained in the reference tensile test.

\section{Conclusion}

A variational method used to identify mechanical properties experimentally was presented in this article. The material properties identified are elastoplastic with a linear kinematic hardening. This identification uses plane displacements given by D.I.C, and the richness and the accuracy of the measurement allow to identify a fine discretized distribution of elastic and plastic parameters. Before applying the method to experimental data, our identification procedure has been verified on the basis of a numerical simulation. To confirm the meaning of this numerical study, a Gaussian white noise was superimposed to the proposed here numerical data.

Both the numerical and the experimental results are very promising, and the method proposed here is readily extensible to isotropic hardening and other plastic models thermomechanically consistent are under the way. The experimental results could be substantially improved by using a temporal filter, which is particularly appropriate in the case of spatial heterogeneities. The method is currently applied to other experimental data, in various configurations.

\section{References}

[1] Wattrisse B, Chrysochoos A, Muracciole JM, Némoz-Gaillard M (2001) Analysis of strain localisation during tensile test by digital image correlation. Journal of Experimental Mechanics 
$41: 29-38$.

[2] Chrysochoos A, Louche H (2000) An infrared image processing to analyse the calorific effects accompanying strain localisation. International Journal of Engineering Science 38:1759-1788.

[3] Grédiac M (2004) The use of full-field measurement methods in composite material characterization: interest and limitations. Composites: Part A 35:751-761.

[4] Geymonat G, Hild F, Pagano S (2002) Identification of elastic parameters by displacement field measurement. C.R. Mécanique 330:403-408.

[5] Meuwissen MHH, Oomens CWJ, Baaijens FPT, Petterson R, Janssen JD (1998) Determination of the elasto-plastic properties of aluminium using a mixed numerical-experimental method. Journal of Materials Processing Technology 75(1-3):204-211.

[6] Grédiac M, Pierron F (2006) Applying the virtual fields method to the identification of elasto-plastic constitutive parameters. International Journal of Plasticity 22(4):602-627.

[7] Roux S, Hild F,Pagano S (2005) A stress scale in full-field identification procedures: a diffuse stress gauge. Eur. J. Mech. A/Solids 24:442-451.

[8] Kohn R,McKenney A (1990) Numerical implementation of a variational method for electric impedance tomography, Inverse Problems 6:389-414.

[9] Lemaitre J, Chaboche JL (1990) Mechanics of solid materials, Cambridge University Press, pp. 205-209.

[10] Germain P (1986) Mécanique, volume 1 of Cours École Polytechnique. Ellipses, chap.VI.4.6., p84.

[11] Simo JC, Hughes TJR (1998) Computational Inelasticity. Springer-Verlag, pp. 126-130.

[12] Tikhonov AN, Arsenin VY (1977) Solutions to Ill-Posed Problems. Winston-Wiley, New York. 